\title{
Searches for new physics in events with multiple leptons at the ATLAS detector
}

\author{
Liv Wiik-Fuchs ${ }^{1}$, a , on behalf of the ATLAS Collaboration \\ ${ }^{1}$ University of Bonn, Nussallee 12, 53115 Bonn, Germany
}

\begin{abstract}
Events containing three or more leptons are an invaluable probe for physics beyond the Standard Model (SM) at the LHC. This paper summarizes a generic search for final states with three or more leptons as well as direct searches for heavy seesaw neutrinos, excited leptons and $W Z$ resonances. All searches were conducted using the data recorded in 2012 in proton-proton collisions at $\sqrt{s}=8 \mathrm{TeV}$ with the ATLAS detector at the LHC.
\end{abstract}

\section{Introduction}

Many theories beyond the Standard Model (SM) predict events with three or more leptons in the final state. These final states benefit from low SM background and high trigger efficiencies at hadron colliders.

Four different searches for exotic physics in final states with three or more leptons, were conducted with the data recorded by the ATLAS detector [1], at the LHC. These searches use the data recorded in 2012 in $p p$ collisions at $\sqrt{s}=8 \mathrm{TeV}$. Firstly a model-independent search for anomalous production of events with three or more leptons in the final state using the entire 2012 dataset of $20.3 \mathrm{fb}^{-1}$ will be presented. Here the final states are separated into different categories depending on lepton flavor and a $Z$ requirement. These are further sub-divided using several variables to gain sensitivity to a broad range of new physics phenomena. This model-independent search is complemented by three model-dependent searches: a search for excited leptons looking for final states with two same-flavor leptons and a photon in $13 \mathrm{fb}^{-1}$; a search for $W Z$ resonances, looking for three leptons in $20.3 \mathrm{fb}^{-1}$; and a search for type-III seesaw neutrinos with four leptons in the final state in $5.8 \mathrm{fb}^{-1}$. Except for the model-independent search which allows up to one hadronically decaying $\tau$ in the final state, only electrons and muons are considered in these analyses. All described analyses require the leptons to be isolated, energetic and consistent with originating from the primary interaction vertex. The backgrounds for these analyses are divided into two categories, namely the irreducible background, consisting of SM processes with three real leptons such as $W Z$ or $Z Z$ production, and the reducible backgrounds, where at least one of the selected leptons originate from a misidentified jet, a semileptonic heavy flavor decay or photon conversion.

\footnotetext{
a e-mail: lwiik@mail.cern.ch
} 


\section{Generic Three Lepton Search}

To cover a broad range of new physics models with multiple-lepton final states, a model-independent search with three or more leptons was performed by the ATLAS experiment [2].

\subsection{Event Selection}

The analysis requires three energetic, isolated leptons originating from the primary interaction, where the two highest-energy leptons are electrons or muons while the third can be a hadronically decaying $\tau$. The events are divided into four separate categories, firstly depending on whether the third lepton is a $\tau$, and secondly whether two of the leptons form a same-flavor opposite-charge pair consistent with originating from an on-shell $Z$ decay. Events with a same-flavor, opposite-charge pair $\left(m_{\ell \ell}<15 \mathrm{GeV}\right)$ are rejected. Further variables are defined to separate the events into 92 different signal regions. These variables are: the transverse sum of the three lepton momenta, $H_{T}^{\text {leptons }}$; the sum of $p_{T}$ of the jets, $H_{T}^{\text {jets }}$; the missing transverse energy, $E_{T}^{\text {miss }}$; the effective mass, $m_{e f f}$, which is the sum of $H_{T}^{\text {leptons }}, E_{T}^{\text {miss }}$ and the $p_{T}$ of the jets in the event; the lowest transverse momentum of the leptons and the number of $b$-tagged jets. As an example for one of the 92 signal regions Fig. 1(a) shows the $H_{T}^{\text {leptons }}$ distribution for the off-Z $3 e / \mu$ category.

\subsection{Background Estimation and Results}

The background composition in this analysis depends heavily on the chosen signal region and category. While categories with three electrons or muons are dominated by irreducible backgrounds, categories with a $\tau$ in the final state are dominated by irreducible backgrounds. The main source of irreducible backgrounds are $W Z, Z Z$ and $t \bar{t}+W / Z$, which are estimated by simulation. The irreducible backgrounds are estimated using a data-driven technique using control regions with loosened lepton identification criteria. No significant excess above Standard Model expectation is observed, therefore upper limits are set at the $95 \%$ confidence-level (CL), on the visible cross-section, using the $C L_{s}$ method, for each of the 92 signal regions. Exemplary Fig. 1(b) shows the observed- and expected95\% CL limit, on the visible cross section as a function of $H_{T}^{\text {leptons }}$, in the different signal categories. Figure 2 shows the deviation of observed yields from expected yields in units of the total uncertainty on the expected yield for all signal regions. For specific model testing purposes the limits in all categories and signal regions, along with the lepton fiducial efficiencies, are available in HEPData ${ }^{1}$.

\section{Type-III Seesaw Neutrinos}

In the so-called seesaw mechanisms the smallness of the neutrino masses is explained by introducing new particles at a high mass scale. This analysis focusses on the type-III seesaw mechanism, which introduces at least two fermionic triplets that generate neutrino masses. The lightest fermionic triplet has two charged heavy leptons denoted $N^{+}$and $N^{-}$, and a neutral heavy lepton $N^{0}$. In the type-III seesaw the heavy states couple to gauge bosons, hence the possible smallness of the Yukawa coupling, does not affect the production cross section of such heavy states at the LHC. Furthermore, the mass of the heavy lepton $\left(m_{N}\right)$ may be of the order of $100 \mathrm{GeV}$, when either the Yukawa couplings are very small or if an inverse seesaw mechanism were at work. At the LHC, the type-III seesaw model manifests itself through pair production of heavy leptons, $N^{0} N^{ \pm}$and $N^{\mp} N^{ \pm}$.

The analysis is further described in [3].

\footnotetext{
${ }^{1}$ http://hepdata.cedar.ac.uk
} 


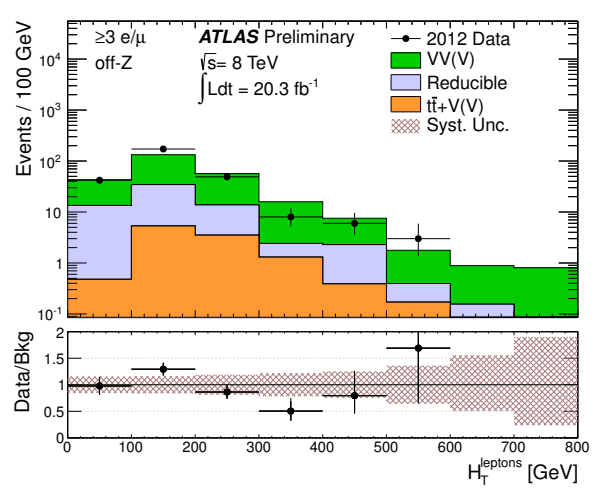

(a)

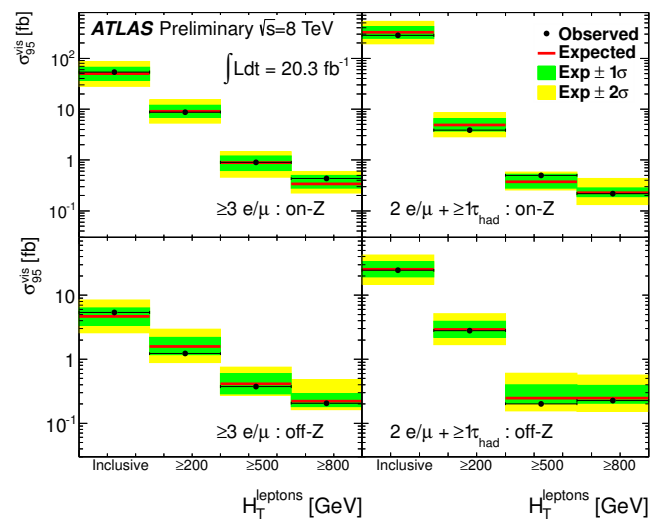

(b)

Figure 1. (a) Distribution of $H_{T}^{\text {leptons }}$ for the off-Z $3 e / \mu$ signal channels. The last bin shows the overflows. The bottom panel shows the ratio of events observed in data to that expected from background sources. (b) Observedand median-expected 95\% CL limit on the visible cross section in the different signal channels, as functions of increasing lower bounds on $H_{T}^{\text {leptons }}$. The $\pm 1 \sigma$ and $\pm 2 \sigma$ uncertainties on the median expected limit are indicated by green and yellow bands, respectively.[2]

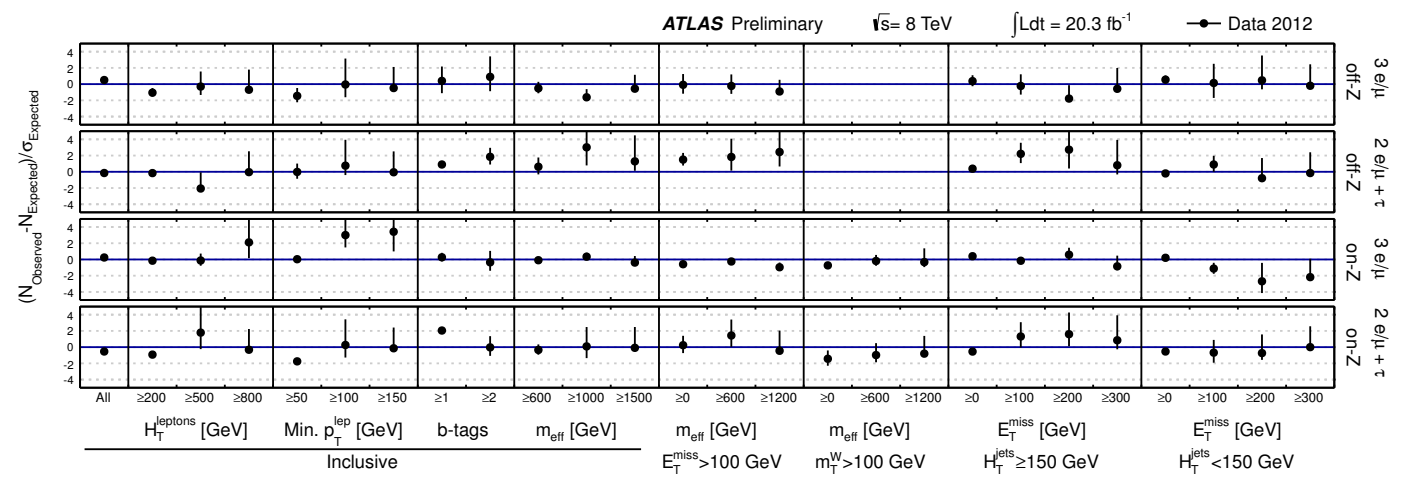

Figure 2. Deviations of observed yields from expected yields, in units of the total uncertainty on the expected yield, for all 92 signal regions under study. [2]

\subsection{Event Selection}

This analysis aims at the direct reconstruction of final states with decays $N^{ \pm} \rightarrow \ell^{ \pm} Z(\ell=e \mu)$, in which the $Z$ decays leptonically. The analysis requires at least four leptons, three attributed to the resonance and a fourth from the opposite side of the event to further discriminate the background. While the transverse momentum of the lepton attributed to the trigger needs to pass $p_{T}>25 \mathrm{GeV}$ the remaining ones are selected with a $p_{T}>10 \mathrm{GeV}$. The resonance $N^{ \pm} \rightarrow Z l^{ \pm}$is reconstructed by selecting a same-flavor opposite-charge lepton-pair with an invariant mass within $10 \mathrm{GeV}$ of the $Z$ 
pole mass. The third lepton is chosen as the closet in the transverse plane to the $Z$ candidate. Events with a second $Z$ candidate are rejected, which efficiently reduces the $Z Z$ background.

\subsection{Background Estimation and Results}

With four leptons in the final state the main background is from $Z Z$ production, followed by minor contributions from $Z+$ jets, $t \bar{t}+W / Z, Z Z Z$ and $Z W W$. All background estimations depend on Monte Carlo simulation. The dominating $Z Z$ background is normalized using a dedicated control region, defined by reverting the veto on the second $Z$ candidate in the event. Figure 3(a) shows the invariant mass distribution of the $Z(\ell \ell) \ell$ system forming the $N^{ \pm}$candidate in the signal region. Since no significant excess above the expected Standard Model background was observed, limits on the product of the cross section times the branching fractions for the process, $\sigma\left(p p \rightarrow N^{ \pm} N^{0}\right) \times B F\left(N^{ \pm} \rightarrow Z l^{ \pm}\right) \times B F\left(N^{0} \rightarrow W^{ \pm} l^{\mp}\right)$, were placed as a function of $m_{N}$. The upper limits at the $95 \% \mathrm{CL}$ are shown in Fig.3(b). With the branching ratio set to the nominal value (magenta curve) the expected limit is $m_{N}>240.3_{-5.6}^{+6.0} \mathrm{GeV}$ and the observed limit is $m_{N}>229.3 \pm 6.2 \mathrm{GeV}$.

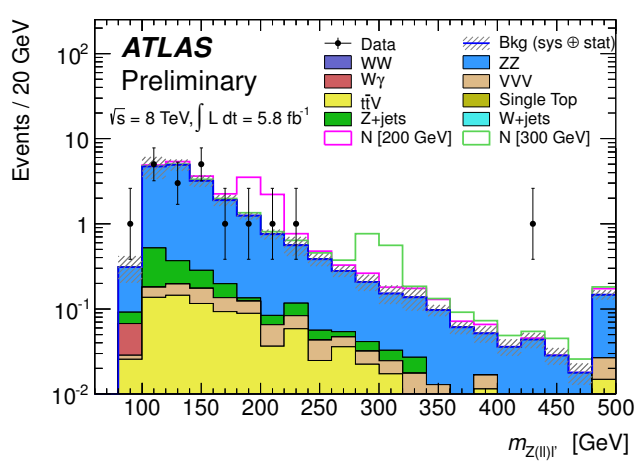

(a)

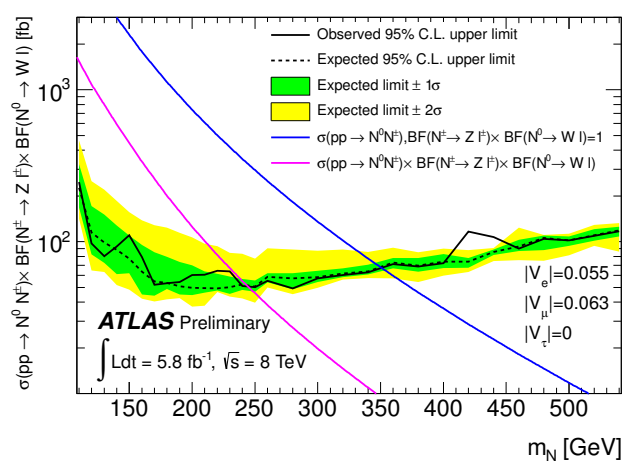

(b)

Figure 3. (a): The $Z(\ell \ell) \ell$ invariant mass distribution, of the $N^{ \pm}$candidates in the signal region, for data (black points) and the expected total background (solid histograms). The rightmost bin in the histogram is the overflow bin. (b): The expected (dashed line) and observed (solid line) exclusion limit at $95 \%$ CL on the cross section times branching fraction as a function of the heavy fermion mass $m_{N}$. [3]

\section{Excited Leptons}

The Standard Model fails to explain both the mass hierarchy and the generational structure of quarks and leptons. Composite models address these questions by hypothesizing that fermions are bound states of more fundamental particles. Excited leptons are a direct consequence of the compositeness, as new interactions of the fermions at the compositeness scale $\Lambda$ can lead to excited states with masses below $\Lambda$. This analysis searches for excited electrons or muons from a four-fermion contact interaction, $q \bar{q} \rightarrow \ell \ell^{*}$, with the excited lepton $\ell^{*}$ decaying via $\ell^{*} \rightarrow \ell \gamma$. The analysis is based on a data sample of $13 \mathrm{fb}^{-1}$.[4] 


\subsection{Event Selection}

This analysis selects two same-flavor opposite-charge leptons and a photon, with stringent criteria on the particle identification and isolation [4]. The photon is required to have a transverse momentum $p_{T}>30 \mathrm{GeV}$, and a good separation to the leptons in the event, $\Delta R(\gamma, \ell)>0.7$. The selected electrons need to pass a $p_{T}$ threshold of $40 \mathrm{GeV}$ for the leading and $30 \mathrm{GeV}$ for the subleading candidate, while both muon candidates are required to have $p_{T}>25 \mathrm{GeV}$. In cases where more than one same-flavor opposite-charge pair is found the one with the highest invariant mass is selected. Furthermore a $Z$ veto is implemented by requiring the invariant mass of the lepton pair to be larger than $110 \mathrm{GeV}$. This efficiently reduces the background from $Z$ decays. Depending on the mass of the excited lepton, two signal regions are defined: $m_{l l \gamma}>m_{\ell^{*}}+150 \mathrm{GeV}$ for $m_{\ell^{*}}<900$, and $m_{l l \gamma}>1050 \mathrm{GeV}$ for $m_{\ell^{*}}>900 \mathrm{GeV}$.

\subsection{Background Estimation and Results}

The dominant background is $Z+\gamma$ production, which is estimated by Monte Carlo simulation. The reducible background, mainly originating from $Z+$ jets events is not well modeled in simulation, and is therefore normalized to data in a control region to account for the misidentification of jets as photons, similarly for the $W+\gamma+j e t s$ background, where jets could be identified as electrons. Due to the small number of events in the simulation remaining after event selection, the background distributions are separately fitted with an exponential function and extrapolated to the high mass region. The resulting dimuon-photon invariant mass distribution is shown in Fig. 4(a), including three different excited muon mass hypothesis at a compositeness scale of $10 \mathrm{TeV}$. No significant excess was observed and thus limits are set on the excited lepton cross section times branching ratio $(\sigma B)$ at 95\% CL. The results are interpreted using a Bayesian approach [5] with a flat prior on $\sigma B$. The limits are presented in Fig. 4(b) for the $\mu^{*}$, in the parameter space defined by the compositeness scale $\Lambda$ and the excited lepton mass, $m_{\ell^{*}}$.

\section{WZ Resonance}

The search for diboson resonances is motivated by the investigation of the source of electroweak symmetry breaking. Many SM extensions such as Little Higgs, many Grand Unified Theories, Technicolor or extra dimensions postulate the existence of a diboson resonance at high masses. The search presented here focuses on a resonant $W Z$ production in the decay channel $\ell v \ell \ell(\ell=e, \mu)$. The results are interpreted in the context of an extended gauge model $W^{\prime}$ (EGM) and a simplified model of heavy vector triplets (HVT). [6]

\subsection{Event Selection}

The analysis requires three leptons with $p_{T}>25 \mathrm{GeV}$ and $E_{T}^{\text {miss }}>25 \mathrm{GeV}$. Events containing a fourth lepton with $p_{T}>20 \mathrm{GeV}$ are rejected. A same-flavor opposite-charge lepton-pair with an invariant mass within $20 \mathrm{GeV}$ around the $Z$ pole mass is selected. For events with several candidates the one closest to the $Z$ pole mass is chosen. Furthermore the rapidity difference between the $W$ and the $Z$, $\Delta y(W, Z)$, is required to be smaller than 1.5. Additionally two signal regions are defined based on the azimuthal angle between the lepton and the missing transverse energy form the $W$ candidate, the highmass region more sensitive to $W^{\prime}$ with $m_{W^{\prime}}>250 \mathrm{GeV}$ with $\Delta \Phi\left(l, E_{T}^{\text {miss }}\right)<1.5 \mathrm{rd}$, and the low-mass region sensitive to $W^{\prime}$ with $m_{W^{\prime}}<250 \mathrm{GeV}$ with $\Delta \Phi\left(l, E_{T}^{\text {miss }}\right)>1.5 \mathrm{rd}$. 


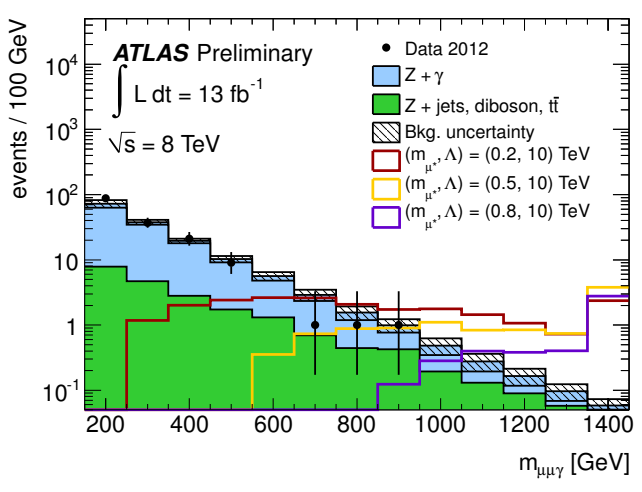

(a)

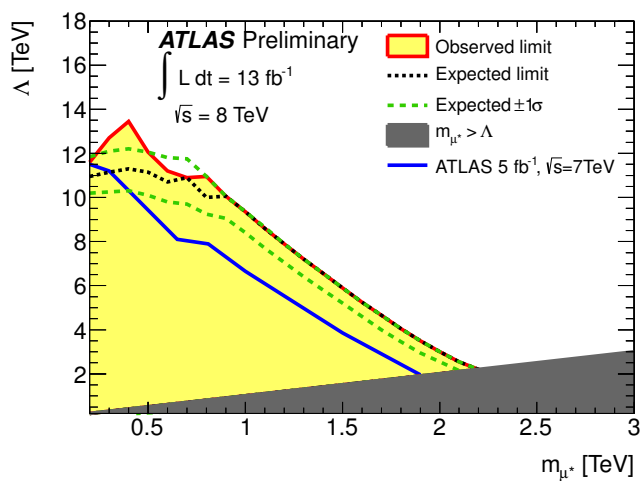

(b)

Figure 4. (a): Dimuon-photon invariant mass of the $\mu^{*}$ channel after the final selection. Both the $Z+j e t s$ and the $Z+\gamma$ backgrounds are fitted with a power law function for the high-mass region. The last bin contains all overflows. Different signal predictions at a compositeness scale of $10 \mathrm{TeV}$ are displayed as well. (b) Exclusion limits on the compositeness scale parameter as a function of the excited lepton mass, $m_{\mu^{*}}$ channel. Regions to the left of the experimental limits (yellow) are excluded at 95\% CL. No limits are set in the dark shaded region where the $\mu^{*}$ mass is greater than the compositeness scale as the model is not applicable.[4]

\subsection{Background Estimation and Results}

The main background in this analysis comes from $W Z$ production, followed by $Z Z$ and $\ell \ell^{\prime}+$ jets production. Both the $W Z$ and $Z Z$ backgrounds are estimated from $M C$ and verified in a control region, which is constructed by reverting the requirement on the rapidity difference to $\Delta y(W, Z)>1.5$. Furthermore the requirement on $\Delta \Phi\left(l, E_{T}^{m i s s}\right)$ is removed. The reducible backgrounds such as $\ell \ell^{\prime}+j e t s$, where a jet is misidentified as a lepton, are estimated in a data-driven approach unsing control regions where some of the lepton identification criteria are relaxed.Figure 5(a) shows the $m_{W Z}$ distribution for the high-mass region, including several signal hypotheses. The extrapolation of the backgrounds to the very-high-mass region was performed using a power law function to fit the SM backgrounds. No excess above the Standard Model was observed in the signal regions. Figure 5(b) shows the observed 95\% CL upper limit on $\sigma(p p \rightarrow X) \times B(X \rightarrow W Z)$ as a function of the signal mass, where $X$ denotes the signal resonance, together with the theoretical cross section of the EGM $W^{\prime}$ and the HVT benchmark model. The observed (expected) exclusion limit of the $W^{\prime}$ mass is 1.52 (1.49) $\mathrm{TeV}$.

\section{Conclusion}

Four multilepton searches for phenomena beyond the Standard Model were presented: Three model dependent searches for type-III seesaw heavy fermions, excited leptons and $W Z$ resonances, and a generic search for events with three or more leptons in the final state. None of these searches showed a significant excess above Standard Model expectation. Therefore limits were set on the models, and on the visible cross section. 


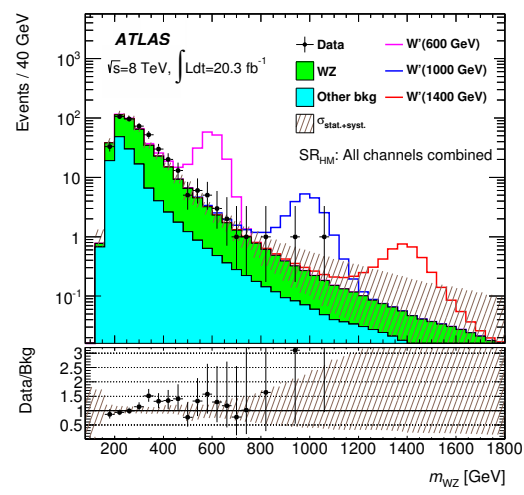

(a)

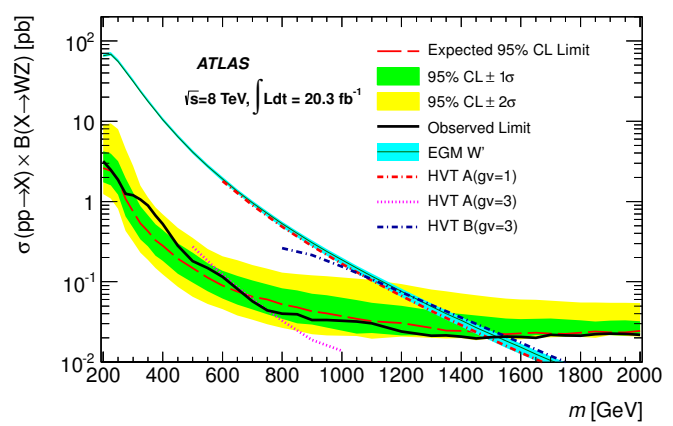

(b)

Figure 5. (a) Observed and predicted $W Z$ invariant mass $\left(m_{W Z}\right)$ distribution for events in the high-mass signal region. The predictions from $W^{\prime}$ samples with masses of $600 \mathrm{GeV}, 1000 \mathrm{GeV}$ and $1400 \mathrm{GeV}$ are also shown. The uncertainty bands on the expected background include both the statistical and systematic uncertainties and the reducible background estimation added in quadrature. Fig. 5(b) observed 95\% CL upper limits on $\sigma(p p \rightarrow$ $X) \times B(X \rightarrow W Z)$ as a function of the signal mass $\mathrm{m}$, where $X$ stands for the signal resonance. The expected limits are also shown together with the \pm 1 and \pm 2 standard deviation uncertainty bands. The theoretical cross sections for the EGM $W^{\prime}$ and the HVT benchmark models are also shown. The uncertainty band around the EGM W' cross section line represents the theoretical uncertainty on the NNLO cross section calculation using ZWPROD [7].

\section{References}

[1] ATLAS Collaboration, "The ATLAS experiment at the CERN Large Hadron Collider", JINST, 3 (2008), S08003.

[2] ATLAS Collaboration, "Search for New Physics in Events with Three Charged Leptons with the ATLAS detector", ATLAS-CONF-2013-070, CERN, Geneva, http : //cds.cern.ch/record/1562898.

[3] ATLAS Collaboration, "Search for Type III Seesaw Model Heavy Leptons in Events with 4 Charged Leptons using 5.8fb-1 of $\sqrt{s}=8 \mathrm{TeV}$ with the Atlas detector ", ATLAS-CONF-2013019, CERN. Geneva http : //cds.cern.ch/record/1525526/.

[4] ATLAS Collaboration, "Search for excited electrons and muons with $13 \mathrm{fb}^{-1}$ of proton-proton collisions at $\sqrt{s}=8 \mathrm{TeV}$ with the ATLAS detector", New J. Phys. 15 (2013), 093011.

[5] A. Caldwell, D. Kollar, K. Kroeninger, "BAT - The Bayesian Analysis Toolkit", Comput. Phys. Commun. 180 (2009) 2197-2209.

[6] ATLAS Collaboration, "Search for $W Z$ resonances in the fully leptonic channel using pp collisions at $\sqrt{s}=8 \mathrm{TeV}$ with the ATLAS detector" , Physics Letters B 737(2014), 223-243.

[7] R. Hamberg, W. van Neerven, T. Matsuura, "A complete calculation of the order $\alpha-s^{2}$ correction to the Drell -Yan K factor", Nucl. Phys. B 359 (1991), 343-405. 